\title{
Some Stability and Convergence of Additive Runge-Kutta Methods for Delay Differential Equations with Many Delays
}

\author{
Haiyan Yuan, ${ }^{1,2}$ Jingjun Zhao, ${ }^{1}$ and Yang $X u^{1}$ \\ ${ }^{1}$ Department of Mathematics, Harbin Institute of Technology, Harbin 150001, China \\ ${ }^{2}$ Department of Mathematics, Heilongjiang Institute of Technology, Harbin 150050, China \\ Correspondence should be addressed to Jingjun Zhao, hit_zjj@hit.edu.cn
}

Received 12 October 2011; Accepted 22 December 2011

Academic Editor: Junjie Wei

Copyright (c) 2012 Haiyan Yuan et al. This is an open access article distributed under the Creative Commons Attribution License, which permits unrestricted use, distribution, and reproduction in any medium, provided the original work is properly cited.

This paper is devoted to the stability and convergence analysis of the additive Runge-Kutta methods with the Lagrangian interpolation (ARKLMs) for the numerical solution of a delay differential equation with many delays. GDN stability and D-Convergence are introduced and proved. It is shown that strongly algebraically stability gives D-Convergence DA, DAS, and ASI stability give GDN stability. Some examples are given in the end of this paper which confirms our results.

\section{Introduction}

Delay differential equations arise in a variety of fields as biology, economy, control theory, electrodynamics (see, e.g., [1-5]). When considering the applicability of numerical methods for the solution of DDEs, it is necessary to analyze the stability of the numerical methods. In the last three decades, many works had dealt with these problems (see, e.g., [6]). For the case of nonlinear delay differential equations, this kind of methodology had been first introduced by Torelli $[7,8]$ and then developed by Bellen and Zennaro [9], Bellen [10], and Zennaro $[11,12]$.

In this paper, we consider the following nonlinear DDEs with $m$ delays:

$$
\begin{gathered}
y^{\prime}(t)=f^{[1]}\left(t, y(t), y\left(t-\tau_{1}\right)\right)+f^{[2]}\left(t, y(t), y\left(t-\tau_{2}\right)\right)+\cdots+f^{[m]}\left(t, y(t), y\left(t-\tau_{m}\right)\right) \quad t \in\left[t_{0}, T\right] \\
y(t)=\varphi(t) \quad t \in\left[t_{0}-\tau, t_{0}\right]
\end{gathered}
$$




$$
\begin{gathered}
z^{\prime}(t)=f^{[1]}\left(t, z(t), z\left(t-\tau_{1}\right)\right)+f^{[2]}\left(t, z(t), z\left(t-\tau_{2}\right)\right)+\cdots+f^{[m]}\left(t, z(t), z\left(t-\tau_{m}\right)\right) \quad t \in\left[t_{0}, T\right] \\
z(t)=\psi(t) \quad t \in\left[t_{0}-\tau, t_{0}\right]
\end{gathered}
$$

where $\tau_{1} \leq \tau_{2} \leq \cdots \leq \tau_{m}=\tau, f^{[v]}:\left[t_{0}, T\right] \times C^{N} \times C^{N} \rightarrow C^{N}, v=1,2, \ldots, m$, and $\varphi, \psi$ : $\left[t_{0}-\tau, t_{0}\right] \rightarrow C^{N}$ are continuous functions such that (1.1) and (1.2) have a unique solution, respectively. Moreover, we assume that there exist some inner product $\langle\cdot, \cdot\rangle$ and the induced norm $\|\cdot\|$ such that

$$
\begin{gathered}
\operatorname{Re}\left\langle f^{[v]}\left(t, y_{1}, u\right)-f^{[v]}\left(t, y_{2}, u\right), y_{1}-y_{2}\right\rangle \leq \sigma_{v}\left\|y_{1}-y_{2}\right\|^{2} \quad v=1,2, \ldots, m, \\
\left\|f^{[v]}\left(t, y, u_{1}\right)-f^{[v]}\left(t, y, u_{2}\right)\right\| \leq r_{v}\left\|u_{1}-u_{2}\right\| \quad v=1,2, \ldots, m,
\end{gathered}
$$

for all $t \in\left[t_{0}, T\right]$, for all $y, y_{1}, y_{2}, u, u_{1}, u_{2} \in C^{N}$, where $\sigma_{v}, r_{v}$ are constants with

$$
0 \leq r_{v} \leq-\sigma_{v}, \quad v=1,2, \ldots, m
$$

Space discretization of some time dependent delay partial differential equations give rise to such delay differential equations containing additive terms with different stiffness properties. In these situations, additive Runge-Kutta (ARK) methods are used. Some recent works about ARK can refer to $[13,14]$. For the additive DDEs (1.1), (1.2), similar to the proof of Theorem 2.1 in [7], it is straightforward to prove that under the conditions (1.3) and (1.4), the analytic solutions satisfy

$$
\|y(t)-z(t)\| \leq \max _{t_{0}-\tau \leq t \leq t_{0}}\|\varphi(t)-\psi(t)\|
$$

To demand the discrete numerical solutions to preserve the stability properties (1.5) of the analytic solutions, Torelli [7] introduced a concept of RN, GRN stability for numerical methods applied to dissipative nonlinear systems of DDEs such as (1.1), which is the straightforward generalization of the well-known concept of BN stability of numerical methods with respect to dissipative systems of ODEs (see also [9]). A disappointing conclusion is, as it is described in [10], that the order of RK methods for DDEs preserving RN-stable properties may not be more than 4 .

To bypass this order barrier, Zhang and Zhou [15] relaxed the RN stability restriction, considered the GDN stability and D-Convergence of (1.1) in the case $m=1$. In 2001, Zhang et al. [16] gave the results of D-Convergence and GDN stability of (1.1) with the vector form. So, the aim of this paper is the study of stability and convergence properties for ARK methods when they are applied to nonlinear delay differential equations with $m$ delays.

\section{The GDN Stability of the Additive Runge-Kutta Method}

In this preparatory section we recall the additive Runge-Kutta method and give out its stability analysis. 
Definition 2.1. An additive Runge-Kutta method with the Lagrangian interpolation (ARKLM) of $s$ stages and $m$ levels for (1.1) is a one-step numerical method which the numerical solution of (1.1) from $y_{n}$ (numerical approximation at $t_{n}$ ) to $y_{n+1}$ (numerical approximation at $t_{n+1}=$ $\left.t_{n}+h\right)$, that is,

$$
\begin{gathered}
y_{n+1}=y_{n}+h \sum_{v=1}^{m} \sum_{j=1}^{s} b_{j}^{[v]} f^{[v]}\left(t_{n}+c_{j} h, y_{j}^{(n)}, \tilde{y}_{j}^{[v](n)}\right) \\
y_{i}^{(n)}=y_{n}+h \sum_{v=1}^{m} \sum_{j=1}^{s} a_{i j}^{[v]} f^{[v]}\left(t_{n}+c_{j} h, y_{j}^{(n)}, \tilde{y}_{j}^{[v](n)}\right) \\
i=1,2, \ldots, s, \quad v=1,2, \ldots, m .
\end{gathered}
$$

Here the coefficients $a_{i j}^{[v]}, b_{j}^{[v]}$, and $c_{j}$ satisfy

$$
\sum_{j=1}^{s} a_{i j}^{[v]}=c_{j}^{[v]}, \quad 0 \leq c_{j} \leq 1, j, v=1,2, \ldots, m,
$$

$t_{n}=t_{0}+n h, y_{n}, y_{j}^{(n)}, \tilde{y}_{j}^{[v](n)}$ are approximations to the analytic solution $y\left(t_{n}\right), y\left(t_{n}+c_{j} h\right)$, $y\left(t_{n}+c_{j} h-\tau_{v}\right)$ of $(1.1)$, respectively, and the argument $\tilde{y}_{j}^{[v](n)}$ is determined by

$$
\tilde{y}_{j}^{[v](n)}= \begin{cases}\varphi\left(t_{n}+c_{j} h-\tau_{v}\right) & t_{n}+c_{j} h-\tau_{v} \leq 0, \\ \sum_{P_{v}=-d}^{r} L_{P_{v}}\left(\delta_{v}\right) y_{j}^{\left(n-m_{v}+P_{v}\right)} & t_{n}+c_{j} h-\tau_{v}>0 .\end{cases}
$$

With $\tau_{v}=\left(m_{v}-\delta_{v}\right), h \delta_{v} \in[0,1)$, integer $m_{v} \geq r+1, r, d \geq 0$, and

$$
L_{P_{v}}\left(\delta_{v}\right)=\prod_{\substack{k=-d \\ k \neq P_{v}}}^{r}\left(\frac{\delta_{v}-k}{P_{v}-k}\right) \quad P_{v}=-d,-d+1, \ldots, r .
$$

We assume $m_{v} \geq r+1$ is to guarantee that no (unknown) values $y_{j}^{(i)}$ with $i \geq n$ are used in the interpolation procedure. In addition, we always put $y_{j}^{(i)}=\varphi\left(t_{n}+c_{j} h\right)$ whenever $n<0$, and $y_{n}=\varphi\left(t_{n}\right)$ whenever $n \leq 0$.

The coefficients of the method may be organized in the Butcher tableau

$$
\begin{array}{l|l|l|l|l}
C & A^{[1]} & A^{[2]} & \cdots & A^{[m]} \\
\hline & b^{[1]^{I}} & b^{[2]^{I}} & \cdots & b^{[m]^{T}}
\end{array}
$$

where $C=\left[c_{1}, c_{2}, \ldots, c_{s}\right]^{T}$ and for $v=1,2, \ldots, m$,

$$
b^{[v]}=\left[b_{1}^{[v]}, b_{2}^{[v]}, \ldots, b_{s}^{[v]}\right], \quad A^{[v]}=\left(a_{i j}^{[v]}\right)_{i, j=1}^{s} .
$$


In order to write (2.1a), (2.1b), and (2.1c) in a more compact way we introduce some notations. The $N \times N$ identity matrix will be denoted by $I_{N}, e=(1,1, \ldots, 1)^{T} \in R^{S}, \widetilde{G}=G \otimes I_{N}$ is the Kronecker product of matrix $G$ and $I_{N}$. For $u=\left(u_{1}, u_{2}, \ldots, u_{S}\right)^{T}, v=\left(v_{1}, v_{2}, \ldots, v_{S}\right)^{T} \in$ $C^{N S}$, we define the inner product and the induced norm in $C^{N S}$ as follows:

$$
\langle u, v\rangle=\sum_{i=1}^{s}\left\langle u_{i}, v_{i}\right\rangle, \quad\|u\|=\sqrt{\sum_{i=1}^{s}\left\|u_{i}\right\|^{2}} .
$$

Moreover, we also adopt that

$$
y^{(n)}=\left[\begin{array}{c}
y_{1}^{(n)} \\
y_{2}^{(n)} \\
\vdots \\
y_{s}^{(n)}
\end{array}\right], \quad \tilde{y}^{[v](n)}=\left[\begin{array}{c}
\tilde{y}_{1}^{[v](n)} \\
\tilde{y}_{2}^{[v](n)} \\
\vdots \\
\tilde{y}_{s}^{[v](n)}
\end{array}\right], \quad f^{[v]}\left(t_{n}, y^{(n)}, \tilde{y}^{[v](n)}\right)=\left[\begin{array}{c}
f^{[v]}\left(t_{n}, y_{1}^{(n)}, \tilde{y}_{1}^{[v](n)}\right) \\
f^{[v]}\left(t_{n}, y_{2}^{(n)}, \tilde{y}_{2}^{[v](n)}\right) \\
\vdots \\
f^{[v]}\left(t_{n}, y_{s}^{(n)}, \tilde{y}_{s}^{[v](n)}\right)
\end{array}\right] .
$$

With the above notation, method (2.1a), (2.1b), and (2.1c) can be written as

$$
\begin{gathered}
y_{n+1}=y_{n}+h \sum_{v=1}^{m} \tilde{b}^{[v]^{T}} f^{[v]}\left(t_{n}, y^{(n)}, \tilde{y}^{[v](n)}\right), \\
y^{(n)}=\tilde{e} y_{n}+h \sum_{v=1}^{m} \tilde{A}^{[v]} f^{[v]}\left(t_{n}, y^{(n)}, \tilde{y}^{[v](n)}\right), \\
\tilde{y}^{[v](n)}= \begin{cases}\tilde{e} \varphi\left(t_{n}+c_{j} h-\tau_{v}\right), & t_{n}+c_{j} h-\tau_{v} \leq t_{0}, \\
\sum_{P_{v}=-d}^{r} L_{P_{v}}\left(\delta_{v}\right) y^{\left(n-m_{v}+P_{v}\right)}, & t_{n}+c_{j} h-\tau_{v}>t_{0} .\end{cases}
\end{gathered}
$$

In 1997, Zhang and Zhou [15] introduced the extension of RN stability to GDN stability as follows.

Definition 2.2. An ARKLM (2.1a), (2.1b), and (2.1c) for DDEs is called GDN stable if, under the conditions (1.3) and (1.4), numerical approximations $y_{n}$ and $z_{n}$ to the solution of (1.1) and (1.2), respectively, satisfy

$$
\left\|y_{n}-z_{n}\right\| \leq C \max _{t_{0}-\tau \leq t<t_{0}}\|\varphi(t)-\psi(t)\|, \quad n \geq 0,
$$


where constant $C>0$ depends only on the method, the parameter $\sigma_{v}, v=1,2, \ldots, m$, and the interval length $T-t_{0}$.

Here, we can see the constant $C$ need not to be less than 1, otherwise the Definition 2.2 is just RN stable in [7].

Definition 2.3. An ARKLM (2.1a), (2.1b), and (2.1c) is called strongly algebraically stable if matrices $M_{\gamma \mu}$ are nonnegative definite, where

$$
M_{\gamma \mu}=B^{[\gamma]} A^{[\mu]}+A^{[\gamma]^{T}} B^{[\mu]}-b^{[\gamma]} b^{[\mu]^{T}}, \quad B^{[\gamma]}=\operatorname{diag}\left(b_{1}^{[\gamma]}, b_{2}^{[\gamma]}, \ldots, b_{s}^{[\gamma]}\right)
$$

for $\mu, \gamma=1,2, \ldots, m$.

Let $\left\{y_{n}, y_{j}^{(n)}, \tilde{y}_{j}^{[1](n)}, \tilde{y}_{j}^{[2](n)}, \ldots, \widetilde{y}_{j}^{[m(n)]}\right\}_{j=1}^{s}$ and $\left\{z_{n}, z_{j}^{(n)}, \widetilde{z}_{j}^{[1](n)}, \widetilde{z}_{j}^{[2](n)}, \ldots, \widetilde{z}_{j}^{[m](n)}\right\}_{j=1}^{s}$ be two sequences of approximations to problems (1.1) and (1.2), respectively. From method (2.1a), (2.1b), and (2.1c) with the samestep size $h$, and write

$$
\begin{gathered}
T_{i}^{(n)}=t_{n}+c_{i} h, \quad U_{i}^{(n)}=y_{i}^{(n)}-z_{i}^{(n)}, \quad \tilde{U}_{i}^{[v](n)}=\tilde{y}_{i}^{[v](n)}-\tilde{z}_{i}^{[v](n)}, \quad U_{0}^{(n)}=y_{n}-z_{n}, \\
Q_{i}^{[v](n)}=h\left[f^{[v]}\left(T_{i}^{(n)}, y_{i}^{(n)}, \tilde{y}_{i}^{[v](n)}\right)-f^{[v]}\left(T_{i}^{(n)}, z_{i}^{(n)}, \tilde{z}_{i}^{[v](n)}\right)\right], \quad i=1,2, \ldots, s, v=1,2, \ldots, m .
\end{gathered}
$$

Then (2.1a) reads

$$
\begin{gathered}
U_{0}^{(n+1)}=U_{0}^{(n)}+\sum_{v=1}^{m} \sum_{i=1}^{s} b_{i}^{[v]} Q_{i}^{[v](n)}, \\
U_{i}^{(n)}=U_{0}^{(n)}+\sum_{v=1}^{m} \sum_{j=1}^{s} a_{i j}^{[v]} Q_{j}^{[v](n)} .
\end{gathered}
$$

Our main results about GDN stability are contained in the following theorem.

Theorem 2.4. Assume ARK method (2.1a) is strongly algebraically stable, and then the corresponding ARKLM (2.1a), (2.1b), and (2.1c) is GDN stable, and satisfies

$$
\left\|y_{n}-z_{n}\right\| \leq \exp \left[-\left(T-t_{0}\right) m \sum_{v=1}^{m} \sigma_{v} L_{0}\right] \max _{t_{0}-\tau \leq t \leq t_{0}}\|\varphi(t)-\psi(t)\|^{2}, \quad n \geq 0
$$

where $L_{0}=\sup _{\delta_{v} \in[0,1)}\left(\sum_{p_{v}=-d}^{r}\left|L_{p_{v}}\left(\delta_{v}\right)\right|\right)^{2}$. 
Proof. From (2.11) we get

$$
\begin{aligned}
\left\|U_{0}^{(n+1)}\right\|^{2}= & \left\langle U_{0}^{(n)}+\sum_{v=1}^{m} \sum_{i=1}^{s} b_{i}^{[v]} Q_{i}^{[v](n)}, U_{0}^{(n)}+\sum_{v=1}^{m} \sum_{i=1}^{s} b_{i}^{[v]} Q_{i}^{[v](n)}\right\rangle \\
= & \left\|U_{0}^{(n)}\right\|^{2}+2 \sum_{v=1}^{m} \sum_{i=1}^{s} b_{i}^{[v]} \operatorname{Re}\left\langle Q_{i}^{[v](n)}, U_{0}^{(n)}\right\rangle+\sum_{u, v=1}^{m} \sum_{i, j=1}^{s} b_{i}^{[u]} b_{j}^{[v]}\left\langle Q_{i}^{[u](n)}, Q_{j}^{[v](n)}\right\rangle \\
= & \left\|U_{0}^{(n)}\right\|^{2}+2 \sum_{v=1}^{m} \sum_{i=1}^{s} b_{i}^{[v]} \operatorname{Re}\left\langle Q_{i}^{[v](n)}, U_{i}^{(n)}-\sum_{v=1}^{m} \sum_{j=1}^{s} a_{i j}^{[v]} Q_{j}^{[v](n)}\right\rangle \\
& +\sum_{u, v=1}^{m} \sum_{i, j=1}^{s} b_{i}^{[u]} b_{j}^{[v]}\left\langle Q_{i}^{[v](n)}, Q_{j}^{[u](n)}\right\rangle \\
= & \left\|U_{0}^{(n)}\right\|^{2}+2 \sum_{v=1}^{m} \sum_{i=1}^{s} b_{i}^{[v]} \operatorname{Re}\left\langle Q_{i}^{[v](n)}, U_{i}^{(n)}\right\rangle \\
& -\sum_{u, v=1}^{m} \sum_{i, j=1}^{s}\left(b_{i}^{[u]} a_{i j}^{[v]}+b_{j}^{[v]} a_{i j}^{[u]}-b_{i}^{[u]} b_{j}^{[v]}\right)\left\langle Q_{i}^{[v](n)}, Q_{j}^{[u](n)}\right\rangle .
\end{aligned}
$$

If the matrices $M_{\gamma \mu}$ are nonnegative definite, then

$$
\left\|U_{0}^{(n+1)}\right\|^{2} \leq\left\|U_{0}^{(n)}\right\|^{2}+2 \sum_{v=1}^{m} \sum_{i=1}^{s} b_{i}^{[v]} \operatorname{Re}\left\langle Q_{i}^{[v](n)}, U_{i}^{(n)}\right\rangle .
$$

Furthermore, by conditions (1.3) and (1.4) and Schwartz inequality we have

$$
\begin{aligned}
\operatorname{Re}\left\langle Q_{j}^{[v](n)}, U_{j}^{(n)}\right\rangle= & h\left\langle f^{[v]}\left(T_{j}^{(n)}, y_{j}^{(n)}, \tilde{y}_{j}^{[v](n)}\right)-f^{[v]}\left(T_{j}^{(n)}, z_{j}^{(n)}, \tilde{z}_{j}^{[v](n)}\right), U_{j}^{(n)}\right\rangle \\
= & h \operatorname{Re}\left\langle f^{[v]}\left(T_{j}^{(n)}, y_{j}^{(n)}, \tilde{y}_{j}^{[v](n)}\right)-f^{[v]}\left(T_{j}^{(n)}, z_{j}^{(n)}, \tilde{y}^{[v](n)}\right), U_{j}^{(n)}\right\rangle \\
& +h \operatorname{Re}\left\langle f^{[v]}\left(T_{j}^{(n)} z_{j}^{(n)}, \tilde{y}^{[v](n)}\right)-f^{[v]}\left(T_{j}^{(n)}, z_{j}^{(n)}, \tilde{z}_{j}^{(n)}\right), U_{j}^{(n)}\right\rangle \\
\leq & h \sigma_{v}\left\|U_{j}^{(n)}\right\|^{2}+h\left\|f^{[v]}\left(T_{j}^{(n)}, z_{j}^{(n)}, \tilde{y}_{j}^{[v](n)}\right)-f^{[v]}\left(T_{j}^{(n)}, z_{j}^{(n)}, \tilde{z}_{j}^{[v](n)}\right)\right\| \cdot\left\|U_{j}^{(n)}\right\| \\
\leq & h \sigma_{v}\left\|U_{j}^{(n)}\right\|^{2}+h r_{v}\left\|\tilde{U}_{j}^{[v](n)}\right\| \cdot\left\|U_{j}^{(n)}\right\| \\
\leq & h \sigma_{v}\left\|U_{j}^{(n)}\right\|^{2}+\frac{1}{2} h r_{v}\left(\left\|\tilde{U}_{j}^{[v](n)}\right\|^{2}+\left\|U_{j}^{(n)}\right\|^{2}\right) .
\end{aligned}
$$

From (1.4), we know $0 \leq r_{v} \leq-\sigma_{v}$. 
Journal of Applied Mathematics

Then, we have

$$
\begin{aligned}
\operatorname{Re}\left\langle Q_{j}^{[v](n)}, U_{j}^{(n)}\right\rangle & \leq h \sigma_{v}\left\|U_{j}^{(n)}\right\|^{2}-\frac{1}{2} h r_{v}\left(\left\|\tilde{U}_{j}^{[v](n)}\right\|^{2}+\left\|U_{j}^{(n)}\right\|^{2}\right) \\
& \leq-\frac{1}{2} h \sigma_{v}\left\|\tilde{U}_{j}^{[v](n)}\right\|^{2} .
\end{aligned}
$$

Substituting (2.16) into (2.14), yields

$$
\left\|U_{0}^{(n+1)}\right\|^{2} \leq\left\|U_{0}^{(n)}\right\|^{2}-h \sum_{v=1}^{m} \sum_{j=1}^{s} \sigma_{v} b_{j}^{[v]}\left\|\tilde{U}_{j}^{[v](n)}\right\|^{2}
$$

In addition, with (2.1c), we have

$$
\begin{aligned}
\left\|\tilde{U}_{j}^{[v](n)}\right\|^{2} & \leq\left[\sum_{P_{v}=-d}^{r}\left|L_{P_{v}}\left(\delta_{v}\right)\right| \cdot\left\|U_{j}^{\left(n-m_{v}+P_{v}\right)}\right\|\right]^{2} \\
& \leq L_{0} \max \left\|U_{j}^{\left(n-m_{v}+P_{v}\right)}\right\|^{2} .
\end{aligned}
$$

Combining (2.17) with (2.18) and using (2.1b) we arrive at

$$
\begin{aligned}
\left\|U_{0}^{(n+1)}\right\|^{2} & \leq\left(1-h \sum_{v=1}^{m} \sum_{j=1}^{s} b_{j}^{[v]} \sigma_{v} L_{0}\right) \max \left\{\left\|U_{0}^{(n)}\right\|^{2}, \max _{\left(j, P_{v}\right) \in E}\left\|U_{j}^{\left(n-m_{v}+P_{v}\right)}\right\|^{2}\right\} \\
& \leq\left(1-h m \sum_{v=1}^{m} \sigma_{v} L_{0}\right) \max \left\{\left\|U_{0}^{(n)}\right\|^{2}, \max _{\left(j, P_{v}\right) \in E}\left\|U_{j}^{\left(n-m_{v}+P_{v}\right)}\right\|^{2}\right\},
\end{aligned}
$$

where $E=\left\{\left(j, P_{v}\right) 1 \leq j \leq s,-d \leq P_{v} \leq r\right\}$.

Similar to (2.19), the inequalities

$$
\left\|U_{i}^{(n)}\right\| \leq\left(1-h m \sum_{v=1}^{m} \sigma_{v} L_{0}\right) \max \left\{\left\|U_{0}^{(n)}\right\|^{2}, \max _{\left(j, P_{v}\right) \in E}\left\|U_{j}^{\left(n-m_{v}+P_{v}\right)}\right\|^{2}\right\} \quad i=1,2, \ldots, s,
$$

follow.

In the following, with the help of inequalities (2.19), (2.20) and induction we will prove the inequalities:

$$
\left\|U_{i}^{(n)}\right\|^{2} \leq\left(1-h m \sum_{v=1}^{m} \sigma_{v} L_{0}\right)^{n+1} \max _{t \leq 0}\|\varphi(t)-\psi(t)\|^{2}, \quad n \geq 0, i=1,2, \ldots, s .
$$

In fact, it is clear from (2.19), (2.20), and $m_{v} \geq r+1$ that

$$
\left\|U_{i}^{(0)}\right\|^{2} \leq\left(1-h m \sum_{v=1}^{m} \sigma_{v} L_{0}\right) \max _{t \leq 0}\|\varphi(t)-\psi(t)\|^{2} \quad i=0,1,2, \ldots, s .
$$


Suppose for $n \leq k(k \geq 0)$ that

$$
\left\|U_{i}^{(n)}\right\|^{2} \leq\left(1-h m \sum_{v=1}^{m} \sigma_{v} L_{0}\right)^{n+1} \max _{t \leq 0}\|\varphi(t)-\psi(t)\|^{2}, \quad i=0,1,2, \ldots, s .
$$

Then from (2.19), (2.20), $m_{v} \geq r+1$, and $\left(1-h \sum_{v=1}^{m} \sigma_{v} L_{0}\right)>1$, we conclude that

$$
\left\|U_{i}^{(k+1)}\right\|^{2} \leq\left(1-h m \sum_{v=1}^{m} \sigma_{v} L_{0}\right)^{k+2} \max _{t_{0}-\tau \leq t \leq t_{0}}\|\varphi(t)-\psi(t)\|^{2}, \quad i=0,1,2, \ldots, s .
$$

This completes the proof of inequalities (2.21). In view of (2.21), we get for $n \geq 0$ that

$$
\begin{aligned}
\left\|U_{0}^{(n)}\right\|^{2} & \leq\left(1-h m \sum_{v=1}^{m} \sigma_{v} L_{0}\right)^{n+1} \max _{t_{0}-\tau \leq t \leq t_{0}}\|\varphi(t)-\psi(t)\|^{2} \\
& \leq \exp \left[-(n+1) m h \sum_{v=1}^{m} \sigma_{v} L_{0}\right] \max _{t_{0}-\tau \leq t \leq t_{0}}\|\varphi(t)-\psi(t)\|^{2} \\
& \leq \exp \left[-\left(T-t_{0}\right) m \sum_{v=1}^{m} \sigma_{v} L_{0}\right] \max _{t_{0}-\tau \leq t \leq t_{0}}\|\varphi(t)-\psi(t)\|^{2} .
\end{aligned}
$$

As a result, we know that method (2.1a), (2.1b), and (2.1c) is GDN stable.

\section{D-Convergence}

In order to study the convergence of numerical methods for DDEs, we have to mention the concept of the convergence for stiff ODEs.

In 1981, Frank et al. [17] introduced the important concept of B-convergence for numerical methods applied to nonlinear stiff initial value problems of ordinary differential equations. Later, there have been rapid developments in the study of B-convergence and a significant number of important results have already been found for Runge-Kutta methods. In fact, B-convergence result is nothing but a realistic global error estimate based on onesided Lipschitz constant [18]. In this section, we start discussing the convergence of ARKLM (2.1a), (2.1b), and (2.1c) for DDEs (1.1) with conditions (1.3) and (1.4). The approach to the derivation of these estimates is similar to that used in [15]. We assume the analytic solution $y(t)$ of (1.1) is smooth enough and its derivatives used later are bounded by

$$
\left\|D^{(i)} y(t)\right\| \leq \widetilde{M}_{i} \quad t \in\left[t_{0}-\tau, T\right]
$$

where

$$
D^{(i)} y(t)= \begin{cases}y^{(i)}(t), & t \in\left(t_{0}+(j-1) h, t_{0}+j h\right), \\ y^{(i)}\left(t_{0}+j h-0\right), & t=t_{0}+j h\end{cases}
$$


Journal of Applied Mathematics

If we introduce some notations

$$
Y^{(n)}=\left[\begin{array}{c}
y\left(t_{n}+c_{1} h\right) \\
y\left(t_{n}+c_{2} h\right) \\
\vdots \\
y\left(t_{n}+c_{s} h\right)
\end{array}\right], \quad \tilde{Y}^{[v](n)}=\left[\begin{array}{c}
y\left(t_{n}+c_{1} h-\tau_{v}\right) \\
y\left(t_{n}+c_{2} h-\tau_{v}\right) \\
\vdots \\
y\left(t_{n}+c_{s} h-\tau_{v}\right)
\end{array}\right]
$$

with the above notations, the local errors in (2.7) can be defined as

$$
\begin{gathered}
y\left(t_{n+1}\right)=y\left(t_{n}\right)+h \sum_{v=1}^{m} \tilde{b}^{[v]^{T}} f^{[v]}\left(t_{n}, Y^{(n)}, \tilde{Y}^{[v](n)}\right)+Q_{n}, \\
Y^{(n)}=\tilde{e} y\left(t_{n}\right)+h \sum_{v=1}^{m} \tilde{A}^{[v]} f^{[v]}\left(t_{n}, Y^{(n)}, \tilde{Y}^{[v](n)}\right)+r_{n},
\end{gathered}
$$

$$
\begin{aligned}
\tilde{Y}^{[v](n)}=\left(\tilde{Y}_{1}^{[v](n)}, \tilde{Y}_{2}^{[v](n)}, \ldots, \tilde{Y}_{s}^{[v](n)}\right)^{T} \text { with } \\
\tilde{Y}_{j}^{[v](n)}= \begin{cases}\varphi\left(t_{n}+c_{j} h-\tau_{v}\right) & t_{n}+c_{j} h-\tau_{v} \leq t_{0}, \\
\sum_{P_{v}=-d}^{r} L_{P_{v}}\left(\delta_{v}\right) y_{j}^{\left(n-m_{v}+P_{v}\right)}+\rho_{j}^{[v](n)} & t_{n}+c_{j} h-\tau_{v}>t_{0} .\end{cases}
\end{aligned}
$$

If we take $\breve{y}_{n}=y\left(t_{n}\right)$ and

$$
\breve{y}^{(n)}=\left[\begin{array}{c}
y\left(t_{n}+c_{1} h\right) \\
y\left(t_{n}+c_{2} h\right) \\
\vdots \\
y\left(t_{n}+c_{s} h\right)
\end{array}\right] \quad \breve{y}^{[v](n)}=\left[\begin{array}{c}
y\left(t_{n}+c_{1} h-\tau_{v}\right) \\
y\left(t_{n}+c_{2} h-\tau_{v}\right) \\
\vdots \\
y\left(t_{n}+c_{s} h-\tau_{v}\right)
\end{array}\right]
$$

Then we can get the perturbed scheme of (2.7)

$$
\begin{gathered}
\tilde{y}_{n+1}=\tilde{y}_{n}+h \sum_{v=1}^{m} \tilde{b}^{[v]^{T}} f^{[v]}\left(t_{n}, \tilde{y}^{(n)}, \breve{y}^{[v](n)}\right)+Q_{n}, \\
\tilde{y}^{(n)}=\tilde{e} \tilde{y}_{n}+h \sum_{v=1}^{m} \tilde{A}^{[v]} f^{[v]}\left(t_{n}, \tilde{y}^{(n)}, \breve{y}^{[v](n)}\right)+r_{n}, \\
\breve{y}^{[v](n)}= \begin{cases}\tilde{e} \varphi\left(t_{n}+c_{j} h-\tau_{v}\right), & t_{n}+c_{j} h-\tau_{v} \leq 0, \\
\sum_{P_{v}=-d}^{r} L_{P_{v}}\left(\delta_{v}\right) \breve{y}^{\left(n-m_{v}+P_{v}\right)}+\rho^{[v](n)}, & t_{n}+c_{j} h-\tau_{v}>0 .\end{cases}
\end{gathered}
$$


With perturbations

$$
Q_{n} \in C^{N}, \quad r_{n}=\left(r_{1}^{(n)^{T}}, r_{2}^{(n)^{T}}, \ldots, r_{s}^{(n)^{T}}\right)^{T}, \quad \rho^{[v](n)}=\left(\rho_{1}^{[v](n)^{T}}, \rho_{2}^{[v](n)^{T}}, \ldots, \rho_{s}^{[v](n)^{T}}\right) \in C^{N S}
$$

According to Taylor formula and the formula in [19, pages 205-212], $Q_{n}, r_{n}$, and $\rho^{[v](n)}$ can be determined, respectively, as following:

$$
\begin{gathered}
Q_{n}=\sum_{l=1}^{P} \frac{h^{l}}{(l-1) !}\left(\frac{1}{l}-\sum_{v=1}^{m} \sum_{j=1}^{s} b_{j}^{[v]} c_{j}^{l-1}\right) D^{(l)} y\left(t_{n}\right)+R_{0}^{(n)}, \\
r_{i}^{(n)}=\sum_{l=1}^{P} \frac{h^{l}}{(l-1) !}\left(\frac{1}{l} c_{i}^{l}-\sum_{v=1}^{m} \sum_{j=1}^{s} a_{i j}^{[v]} c_{j}^{l-1}\right) D^{(l)} y\left(t_{n}\right)+R_{i}^{(n)}, \\
\rho_{i}^{[v](n)}=\frac{h^{q+1}}{(q+1) !} \prod_{P_{v}=-d}^{r}\left(\delta_{v}-P_{v}\right) D^{(q+1)} y\left(\xi_{i}^{(n)}\right), \\
\xi_{i}^{(n)} \in\left(t_{n-m_{v}-d}+c_{i} h, t_{n-m_{v}+r}+c_{i} h\right),
\end{gathered}
$$

where $q=d+r, R_{i}^{(n)}$, and $\xi_{i}^{(n)}$ satisfy $\left\|R_{i}^{(n)}\right\| \leq \widehat{M}_{i} h^{i+1}, i=0,1,2, \ldots, s, h \in\left(0, h_{0}\right], h_{0}$ depends only on the method, and $\widetilde{M}_{i}(i=0,1,2, \ldots, s)$ depends only on the method and some $\widetilde{M}_{i}$ in (3.2).

Combining (2.7) with (3.6a), (3.6b), and (3.6c) yields the following recursion scheme for the $\varepsilon_{0}^{(n+1)}=\breve{y}_{n+1}-y_{n+1}$ :

$$
\begin{gathered}
\varepsilon_{0}^{(n+1)}=\varepsilon_{0}^{(n)}+h \sum_{v=1}^{m} \tilde{b}^{[v]^{T}}\left\{f^{[v]}\left(t_{n}, y_{n}, \breve{\breve{y}}^{[v](n)}\right)-f^{[v]}\left(t, y_{n}, \tilde{y}^{[v](n)}\right)+g^{[v](n)} \varepsilon_{n}\right\}+Q_{n} \\
\varepsilon_{n}=\tilde{e} \varepsilon_{0}^{(n)}+h \sum_{v=1}^{m} \tilde{A}^{[v]}\left\{f^{[v]}\left(t_{n}, y_{n}, \breve{\widetilde{y}}^{[v](n)}\right)-f^{[v]}\left(t, y_{n}, \tilde{y}^{[v](n)}\right)+g^{[v](n)} \varepsilon_{n}\right\}+r_{n},
\end{gathered}
$$

where $\varepsilon_{0}^{(n+1)}=\breve{y}_{n+1}-y_{n+1}, \varepsilon_{n}=\left(\varepsilon_{1}^{(n)^{T}}, \varepsilon_{2}^{(n)^{T}}, \ldots, \varepsilon_{s}^{(n)^{T}}\right)^{T}=\breve{y}^{(n)}-y^{(n)}$ and $g_{i}^{[v](n)}=$ $\int_{0}^{1} f_{2}\left(t_{n}+c_{i} h, y_{i}^{(n)}+\theta\left(\tilde{y}_{i}^{(n)}-y_{i}^{(n)}\right), \breve{y}^{[v](n)}\right) d \theta, i=1,2, \ldots, s, f_{2}(t, u, v)$ is the Jacobian matrix $(\partial f(t, u, v) / \partial u)\left(t \in R, u, v \in C^{N}\right)$. 
Assume that $\left(\tilde{I}_{s}-h \sum_{v=1}^{m} \tilde{A}^{[v]} g^{[v](n)}\right)$ is regular, from (3.9), we can get

$$
\begin{aligned}
\varepsilon_{0}^{(n+1)}= & {\left[I_{N}+h \sum_{v=1}^{m} \tilde{b}^{[v]^{T}}\left(\tilde{I}_{S}-h \sum_{v=1}^{m} \tilde{A}^{[v]} g^{[v](n)}\right)^{-1} \tilde{e}^{[v](n)}\right] \varepsilon_{0}^{(n)} } \\
& +h \sum_{v=1}^{m} \tilde{b}^{[v]^{T}} g^{[v](n)}\left[\tilde{I}_{S}-h \sum_{v=1}^{m} \tilde{A}^{[v]} g^{[v](n)}\right]^{-1} r_{n} \\
& +h \sum_{v=1}^{m} \tilde{b}^{[v]^{T}}\left[\tilde{I}_{S}+\left(\tilde{I}_{S}-h \sum_{v=1}^{m} \tilde{A}^{[v]} g^{[v](n)}\right)^{-1}\left(h \sum_{v=1}^{m} \tilde{A}^{[v]} g^{[v](n)}\right)\right] \\
& \cdot\left[f^{[v]}\left(t_{n}, y^{(n)}, \breve{y}^{[v](n)}\right)-f^{[v]}\left(t, y^{(n)}, \tilde{y}^{[v](n)}\right)\right]+Q_{n} .
\end{aligned}
$$

Now, we introduce the concept of D-Convergence from [15].

Definition 3.1. An ARKLM (2.1a), (2.1b), and (2.1c) with $y_{n}=y\left(t_{n}\right)(n \leq 0), y_{i}^{(n)}=y\left(t_{n}+c_{i} h\right)$ $(n<0)$ and $\tilde{y}_{i}^{[v](n)}=y\left(t_{n}+c_{i} h-\tau_{v}\right)(n<0)$ is called D-Convergence of order $p$ if this method, when applied to any given DDEs (1.1) subject to (1.3) and (1.4), produces an approximation sequence $y_{n}$, and the global error satisfies a bound of the form

$$
\left\|y\left(t_{n}\right)-y_{n}\right\| \leq C\left(t_{n}\right) h^{P}, \quad h \in\left(0, h_{0}\right]
$$

where the maximum stepsize $h_{0}$ depends on characteristic parameter $\sigma_{v}$ and the method, the function $C(t)$ depends only on some $\widetilde{M}_{i}$ in (3.2), delay $\tau_{v}$, characteristic parameters $\sigma_{v}, r_{v}$ and the method, $v=1,2, \ldots, m$.

Definition 3.2. The ARKLM (2.1a), (2.1b), and (2.1c) is said to be DA stable if the matrix $\left(I_{s}-\sum_{v=1}^{m} A^{[v]} \xi\right)$ is regular for $\xi \in C^{-}:=\{\xi \in C \mid \operatorname{Re} \xi \leq 0\}$ and $\left|R_{i}(\xi)\right| \leq 1$, for all $\xi \in C^{-}$, $i=0,1, \ldots, s$, where

$$
\begin{gathered}
R_{i}\left(\varepsilon_{1}\right)=1+\sum_{v=1}^{m} A_{i}^{[v]} \varepsilon_{1}\left(I_{s}-\sum_{v=1}^{m} A^{[v]} \xi\right)^{-1} e, \\
A_{0}^{[v]}=b^{[v]}, \quad A_{i}^{[v]}=\left(a_{i 1}^{[v]}, a_{i 2}^{[v]}, \ldots, a_{i s}^{[v]}\right)^{T}, \quad i=0,1, \ldots, s .
\end{gathered}
$$

Definition 3.3. The ARKLM (2.1a), (2.1b), and (2.1c) is said to be ASI stable if the matrix $\left(I_{s}-\sum_{v=1}^{m} A^{[v]} \xi\right)$ is regular for $\xi \in C^{-}$, and $\left(I_{s}-\sum_{v=1}^{m} A^{[v]} \xi\right)^{-1}$ is uniformly bounded for $\xi \in C^{-}$. 
Definition 3.4. The ARKLM (2.1a), (2.1b), and (2.1c) is said to be DAS stable if the matrix $\left(I_{s}-\sum_{v=1}^{m} A^{[v]} \xi\right)$ is regular for $\xi \in C^{-}$, and $\sum_{v=1}^{m} A_{i}^{[v]^{T}} \xi\left(I_{s}-\sum_{v=1}^{m} A^{[v]} \xi\right)^{-1}(i=0,1, \ldots, s)$ is uniformly bounded for $\xi \in C^{-}$.

Lemma 3.5. Suppose the ARKLM is DA, DAS, and ASI stable, then there exist positive constants $h_{0}, \gamma_{1}, \gamma_{2}, \gamma_{3}$, which depend only on the method and the parameter $\sigma_{v}, r_{v}$, such that

$$
\begin{gathered}
\left\|\tilde{I}_{s}-\sum_{v=1}^{m} \tilde{A}^{[v]} \xi\right\| \leq \gamma_{1} \\
\left\|I_{N}+\sum_{v=1}^{m} \tilde{A}_{i}^{[v]^{T}} \xi\left(\tilde{I}_{S}-\sum_{v=1}^{m} \tilde{A}^{[v]} \xi\right)^{-1} \tilde{e}\right\| \leq 1+\gamma_{2} h \\
\left\|\sum_{v=1}^{m} \tilde{A}_{i}^{[v]^{T}} \xi\left(\tilde{I}_{s}-\sum_{v=1}^{m} \tilde{A}^{[v]} \xi\right)^{-1} v\right\| \leq \gamma_{3}\|v\|, \quad v \in C^{N S}, \\
h \in\left(0, h_{0}\right], \quad i=0,1,2, \ldots, s .
\end{gathered}
$$

Proof. This lemma can be proved in similar way as that of the one in [20, Lemmas 3.5-3.7].

Theorem 3.6. Suppose the ARKLM (2.1a), (2.1b), and (2.1c) is DA, DAS, and ASI stable, then there exist positive constants $h_{0}, \gamma_{3}, \gamma_{4}, \gamma_{5}$, which depend only on the method and the parameters $\sigma_{v}$, $r_{v}$, such that for $h \in\left(0, h_{0}\right]$

$$
\left\|\varepsilon_{i}^{(n)}\right\| \leq\left\{\begin{array}{c}
1+h \gamma_{4} \max \left\{\left\|\varepsilon_{0}^{(n+1)}\right\|, \max _{\left(i, p_{v}\right)}\left\|\varepsilon_{i}^{\left(n-1-m_{v}+p_{v}\right)}\right\|\right\} \\
+h \gamma_{5} \max _{1 \leq i \leq s}\left\|\rho_{i}^{(n-1)}\right\|+\left\|Q_{n-1}\right\|+\gamma_{3}\left\|\gamma_{n-1}\right\|, \quad i=0, \\
1+h \gamma_{4} \max _{1}\left\{\left\|\varepsilon_{0}^{(n+1)}\right\|, \max _{\left(i, p_{v}\right)}\left\|\varepsilon_{i}^{\left(n-m_{v}+p_{v}\right)}\right\|\right\} \\
+h \gamma_{5} \max _{1 \leq i \leq s}\left\|\rho_{i}^{(n)}\right\|+\left\|Q_{n}\right\|+\gamma_{3}\left\|\gamma_{n}\right\|, \quad i=1,2, \ldots, s,
\end{array}\right.
$$

where

$$
\begin{gathered}
\varepsilon_{0}^{(n)}=\breve{y}_{n}-y_{n}, \quad \varepsilon_{i}^{(n)}=\breve{y}_{i}^{(n)}-y_{i}^{(n)}, \\
E=\left\{\left(i, p_{v}\right) \mid, 1 \leq i \leq s,-d \leq p_{v} \leq \gamma\right\}, \quad E_{m}=\{(j, v) \mid 0 \leq j \leq s, 1 \leq v \leq m\} .
\end{gathered}
$$


Journal of Applied Mathematics

Proof. Using (3.10) and Lemma 3.5, for $h \in\left(0, h_{0}\right]$, we obtain that

$$
\begin{aligned}
& \left\|\varepsilon_{0}^{(n+1)}\right\| \leq\left(1+\gamma_{2} h\right)\left\|\varepsilon_{0}^{(n)}\right\|+h \gamma_{3}\left\|\sum_{v=1}^{m} A^{[v]}\left[f^{[v]}\left(t_{n}, y^{(n)}, \breve{y}^{[v](n)}\right)-f^{[v]}\left(t_{n}, y^{(n)}, \tilde{y}^{[v](n)}\right)\right]\right\| \\
& +h\left\|\sum_{v=1}^{m} \tilde{b}^{[v]^{T}}\left[f^{[v]}\left(t_{n}, y^{(n)}, \breve{\breve{y}}^{[v](n)}\right)-f^{[v]}\left(t_{n}, y^{(n)}, \tilde{y}^{[v](n)}\right)\right]\right\|+r_{3}\left\|r_{n}\right\|+\left\|Q_{n}\right\| \\
& \leq\left(1+\gamma_{2} h\right)\left\|\varepsilon_{0}^{(n)}\right\|+\gamma_{3}\left\|r_{n}\right\|+\left\|Q_{n}\right\| \\
& +h \gamma_{3} \sum_{v=1}^{m} \sqrt{\sum_{i=1}^{s}\left\|\sum_{j=1}^{s} a_{i j}^{[v]}\left[f^{[v]}\left(t_{n}, c_{j} h, y_{j}^{(n)}, \breve{y}_{j}^{[v](n)}\right)-f^{[v]}\left(t_{n}, c_{j} h, y_{j}^{(n)}, \tilde{y}_{j}^{[v](n)}\right)\right]\right\|^{2}} \\
& +h \sum_{v=1}^{m}\left\|\sum_{j=1}^{s} b_{j}^{[v]}\left[f^{[v]}\left(t_{n}+c_{j} h, y_{j}^{(n)}, \breve{\breve{y}}_{j}^{[v](n)}\right)-f^{[v]}\left(t_{n}+c_{j} h, y_{j}^{(n)}, \tilde{y}^{[v](n)}\right)\right]\right\| \\
& \leq\left(1+\gamma_{2} h\right)\left\|\varepsilon_{0}^{(n)}\right\|+\gamma_{3}\left\|\gamma_{n}\right\|+\left\|Q_{n}\right\|+h \gamma_{3} \sum_{v=1}^{m} \sqrt{\sum_{i=1}^{s}\left[\sum_{j=1}^{s}\left|a_{i j}^{[v]}\right| \gamma_{v}\left\|\breve{y}_{j}^{[v](n)}-\tilde{y}_{j}^{[v](n)}\right\|\right]^{2}} \\
& +h \sum_{v=1}^{m} \sum_{j=1}^{s}\left|b_{j}^{(v)}\right| \gamma_{v}\left\|\breve{\widetilde{y}}_{j}^{[v](n)}-\tilde{y}_{j}^{[v](n)}\right\| \\
& \leq\left(1+\gamma_{2} h\right)\left\|\varepsilon_{0}^{(n)}\right\|+\gamma_{3}\left\|\gamma_{n}\right\|+\left\|Q_{n}\right\| \\
& +h \sum_{v=1}^{m} \gamma_{v}\left(\gamma_{3} \sqrt{\sum_{i=1}^{s}\left(\sum_{j=1}^{s}\left|a_{i j}^{[v]}\right|\right)^{2}}+\sum_{j=1}^{s}\left|b_{j}^{[v]}\right|\right) \max _{(j, v) \in E_{m}}\left\|\widetilde{y}_{j}^{[v](n)}-\tilde{y}_{j}^{[v](n)}\right\| .
\end{aligned}
$$

Moreover, it follows from (2.7) and (3.6c) that

$$
\left\|\breve{y}_{j}^{[v](n)}-\tilde{y}_{j}^{[v](n)}\right\| \leq \sup _{\delta_{v} \in[0,1)} \sum_{P_{v}=-d}^{r}\left|L_{P_{v}}\left(\delta_{v}\right)\right| \max _{-d \leq P_{v} \leq r}\left\|\varepsilon_{j}^{\left(n-m_{v}+P_{v}\right)}\right\|+\left\|\rho_{j}^{[v](n)}\right\| .
$$

Substituting (3.17) in (3.16), we get

$$
\begin{aligned}
\left\|\varepsilon_{0}^{(n+1)}\right\| \leq & \left(1+\gamma_{4}^{(0)} h\right) \max _{\left(j, P_{v}\right) \in E}\left\{\left\|\varepsilon_{0}^{(n)}\right\|, \max \left\|\varepsilon_{j}^{\left(n-m_{v}+P_{v}\right)}\right\|\right\} \\
& +h \gamma_{5}^{(0)} \max _{(j, v) \in E_{m}}\left\|\rho_{j}^{[v](n)}\right\|+\left\|Q_{n}\right\|+\gamma_{3}\left\|r_{n}\right\| \quad h \in\left(0, h_{0}\right]
\end{aligned}
$$

where $\gamma_{4}^{(0)}=\gamma_{2}+\gamma_{s}^{(0)} \sup _{\delta_{v} \in[0,1)} \sum_{P_{v}=-d}^{r}\left|L_{P_{v}}\left(\delta_{v}\right)\right|, \gamma_{5}^{(0)}=\sum_{v=1}^{m} \gamma_{v}\left(\gamma_{3} \sqrt{\sum_{i=1}^{s}\left(\sum_{j=1}^{s}\left|a_{i j}^{[v]}\right|\right)^{2}}+\right.$ $\left.\sum_{j=1}^{s}\left|b_{j}^{[v]}\right|\right)$. 
By Lemma 3.5, similar to (3.18), the inequalities

$$
\begin{aligned}
\left\|\varepsilon_{i}^{(n)}\right\| \leq & \left(1+h \gamma_{4}^{(i)}\right) \max _{\left(j, P_{v}\right) \in E}\left\{\left\|\varepsilon_{0}^{(n)}\right\|, \max _{\left(j, P_{v}\right) \in E}\left\|\varepsilon_{j}^{\left(n-m_{v}+P_{v}\right)}\right\|\right\} \\
& +h \gamma_{5}^{(i)} \max _{(j, v) \in E_{m}}\left\|\rho_{j}^{[v](n)}\right\|+\left\|Q_{n}\right\|+\gamma_{3}\left\|r_{n}\right\| \quad i=1,2, \ldots, s, h \in\left(0, h_{0}\right]
\end{aligned}
$$

follow, where

$$
\gamma_{4}^{(i)}=\gamma_{2}+\gamma_{s}^{(i)} \sup _{\delta_{v} \in[0,1)} \sum_{P_{v}=-d}^{r}\left|L_{P_{v}}\left(\delta_{v}\right)\right|, \quad \gamma_{5}^{(i)}=\sum_{v=1}^{m}\left[\gamma_{v}\left(\gamma_{3} \sqrt{\sum_{i=1}^{s}\left(\sum_{j=1}^{s}\left|a_{i j}^{[v]}\right|\right)^{2}}+\sum_{j=1}^{s}\left|a_{i j}^{[v]}\right|\right] .\right.
$$

Setting $\gamma_{4}=\max \left\{\gamma_{4}^{(i)} \mid 0 \leq i \leq s\right\}, \gamma_{5}=\max \left\{\gamma_{5}^{(i)} \mid 0 \leq i \leq s\right\}$, and combining (3.18) with (3.19), we immediately obtain the conclusion of this theorem.

Now, we turn to study the convergence of ARKLM (2.1a), (2.1b), and (2.1c) for (1.1). It is always assumed that the analytic solution $y(t)$ of $(1.1)$ is smooth enough on each internal of the form $\left(t_{0}+(j-1) h, t_{0}+j h\right)(j$ is a positive integer) as (3.2) defined.

Theorem 3.7. Assume ARKLM (2.1a), (2.1b), and (2.1c) with stage order $p$ is DA, DAS, and ASI stable, then the ARKLM (2.1a), (2.1b), and (2.1c) is D-Convergent of order $\min \{p, q+1\}$, where $q=d+r$.

Proof. By Theorem 3.6, we have for $h \in\left(0, h_{0}\right]$

$$
\left\|\varepsilon_{i}^{(n)}\right\| \leq \begin{cases}\left(1+h \gamma_{4}\right) \max \left\{\left\|\varepsilon_{0}^{(n-1)}\right\|, \max _{\left(i, P_{v}\right) \in E}\left\|\varepsilon_{i}^{\left(n-1-m_{v}+P_{v}\right)}\right\|\right\}+T_{1} h^{P+1}+T_{2} h^{q+2}, & i=0, \\ \left(1+h \gamma_{4}\right) \max \left\{\left\|\varepsilon_{0}^{(n)}\right\|, \max _{\left(i, P_{v}\right) \in E}\left\|\varepsilon_{i}^{\left(n-m_{v}+P_{v}\right)}\right\|\right\}+T_{1} h^{P+1}+T_{2} h^{q+2}, & i=1,2, \ldots, s,\end{cases}
$$

where

$$
T_{1}=\widehat{M}_{0}+\gamma_{3} \sqrt{\sum_{i=1}^{s} \widehat{M}_{i}^{2}}, \quad T_{2}=\frac{\gamma_{5}}{(q+1) !} \sum_{v=1}^{m} \prod_{P_{v}=-d}^{r}\left|\delta_{v}-P_{v}\right| M_{q+1} .
$$

It follows from an induction to (3.21) for $n$ that

$$
\left\|\varepsilon_{i}^{(n)}\right\| \leq \begin{cases}\sum_{j=0}^{n}\left(1+h \gamma_{4}\right)^{j}\left(T_{1} h^{p+1}+T_{2} h^{q+2}\right), & i=0, \\ \sum_{j=0}^{n+1}\left(1+h \gamma_{4}\right)^{j}\left(T_{1} h^{p+1}+T_{2} h^{q+2}\right), & i=1,2, \ldots, s, h \in\left(0, h_{0}\right] .\end{cases}
$$


Journal of Applied Mathematics

Hence, for $h \in\left(0, h_{0}\right]$, we arrive at

$$
\begin{aligned}
\left\|y\left(t_{n}\right)-y_{n}\right\| & =\left\|\varepsilon_{0}^{(n)}\right\| \leq \sum_{j=0}^{n}\left(1+h \gamma_{4}\right)^{j}\left(T_{1} h^{p+1}+T_{2} h^{q+2}\right) \\
& =\frac{\left(1+h \gamma_{4}\right)^{n+1}-1}{h \gamma_{4}}\left(T_{1} h^{p+1}+T_{2} h^{q+2}\right) \\
& \leq \frac{\exp \left[(n+1) h \gamma_{4}\right]-1}{\gamma_{4}}\left(T_{1} h^{p}+T_{2} h^{q+1}\right) \\
& \leq c(t) h^{\min \{p, q+1\}},
\end{aligned}
$$

where

$$
c(t)= \begin{cases}\frac{\exp \left[\left(t-t_{0}\right) \gamma_{4}\right] \exp \left(h_{0} \gamma_{4}\right)-1}{\gamma_{4}}\left(T_{1}+T_{2} h_{0}^{q+1-p}\right), & p \leq q, \\ \frac{\exp \left[\left(t-t_{0}\right) \gamma_{4}\right] \exp \left(h_{0} \gamma_{4}\right)-1}{\gamma_{4}}\left(T_{1} h_{0}^{p-q-1}+T_{2}\right), & p>q .\end{cases}
$$

Therefore, the ARKLM (2.1a), (2.1b), and (2.1c) is D-Convergent of order $\min \{p, q+1\},(q=$ $r+d)$.

\section{Some Examples}

In this final section we give some ARK methods to illustrate our theory in this paper.

Example 4.1. The two-stage additive RK method

$$
\begin{array}{c|cc|cc}
0 & \frac{1}{2} & -\frac{1}{2} & 0 & 0 \\
1 & \frac{1}{2} & \frac{1}{2} & 0 & 1 \\
\hline & \frac{1}{2} & \frac{1}{2} & 0 & \frac{1}{2}
\end{array}
$$

with order one is GDN stable by Theorem 2.4, since

$$
M^{11}=\left[\begin{array}{cc}
\frac{1}{4} & -\frac{1}{4} \\
-\frac{1}{4} & \frac{1}{4}
\end{array}\right], \quad M^{12}=\left[\begin{array}{ll}
0 & 0 \\
0 & \frac{1}{2}
\end{array}\right], \quad M^{21}=\left[\begin{array}{ll}
0 & 0 \\
0 & \frac{1}{2}
\end{array}\right], \quad M^{22}=\left[\begin{array}{ll}
0 & 0 \\
0 & \frac{3}{4}
\end{array}\right]
$$

are nonnegative definite. 
Moreover, the method (4.1) is also DA, ASI, and DAS stable, since

$$
\begin{gathered}
I_{2}-\sum_{V=1}^{2} A^{[V]} \xi=\left[\begin{array}{cc}
1-\frac{1}{2} \xi & \frac{1}{2} \xi \\
-\frac{1}{2} \xi & -\frac{3}{2} \xi
\end{array}\right] \text { is regular for } \xi \in C^{-}:=\{\xi \in C \mid \operatorname{Re} \xi \leq 0\} \\
\left(I_{2}-\sum_{V=1}^{2} A^{[V]} \xi\right)=\frac{\left[\begin{array}{ll}
-(3 / 2) \xi(1 / 2) \xi \\
-(1 / 2) \xi 1-(1 / 2) \xi
\end{array}\right]}{\left(\xi^{2}-(3 / 2) \xi\right)} \\
\sum_{V=1}^{2} A_{1}^{[V]^{T}} \xi\left(I_{2}-\sum_{V=1}^{2} A^{[V]} \xi\right)^{-1}=\frac{\left[(1 / 2) \xi^{2},-(1 / 2) \xi^{2}+(1 / 2) \xi\right]}{\left(\xi^{2}-(3 / 2) \xi\right)} \\
\sum_{V=1}^{2} A_{2}^{[V]^{T}} \xi\left(I_{2}-\sum_{V=1}^{2} A^{[V]} \xi\right)^{-1}=\frac{\left[-(3 / 2) \xi^{2},(3 / 2) \xi-(1 / 2) \xi^{2}\right]}{\left(\xi^{2}-(3 / 2) \xi\right)}
\end{gathered}
$$

and (4.1), (4.3a)-(4.3d) are uniformly bounded for $\xi \in \mathrm{C}^{-}$,

$$
\begin{gathered}
R_{1}(\xi)=1+\sum_{V=1}^{2} A_{1}^{[V]^{T}} \xi\left(I_{2}-\sum_{V=1}^{2} A^{[V]} \xi\right)^{-1} e=1+\frac{(1 / 2) \xi}{\xi^{2}-(3 / 2) \xi}=\frac{\xi^{2}-\xi}{\xi^{2}-(3 / 2) \xi^{\prime}} \\
R_{2}(\xi)=1+\sum_{V=1}^{2} A_{2}^{[V]^{T}} \xi\left(I_{2}-\sum_{V=1}^{2} A^{[V]} \xi\right)^{-1} e=\frac{-\xi^{2}+(3 / 2) \xi}{\xi^{2}-(3 / 2) \xi}=1,
\end{gathered}
$$

and (4.3e)-(4.3f) satisfy that $\left|R_{i}(\xi)\right| \leq 1$ for $\xi \in C^{-}, i=1,2$.

By Theorem 3.7, we know that the ARKLM (2.1a), (2.1b), and (2.1c) corresponding to the method (4.1) is D-Convergent of order one.

Example 4.2. The two-stage additive RK method

$$
\begin{array}{l|ll|ll}
1 & 1 & 0 & 1 & 0 \\
1 & \frac{1}{2} & \frac{1}{2} & 0 & 1 \\
\hline & \frac{1}{2} & \frac{1}{2} & 0 & 1
\end{array}
$$


Journal of Applied Mathematics

is strongly algebraically stable, since

$$
M^{11}=\left[\begin{array}{cc}
\frac{3}{4} & 0 \\
0 & \frac{1}{4}
\end{array}\right], \quad M^{12}=\left[\begin{array}{cc}
\frac{1}{2} & 0 \\
0 & \frac{1}{2}
\end{array}\right], \quad M^{21}=\left[\begin{array}{cc}
\frac{1}{2} & 0 \\
0 & \frac{1}{2}
\end{array}\right], \quad M^{22}=\left[\begin{array}{ll}
0 & 0 \\
0 & 1
\end{array}\right]
$$

are nonnegative definite.

Moreover, the method (4.4) is also DA, ASI, and DAS stable. Since

$$
\begin{gathered}
I_{2}-\sum_{V=1}^{2} A^{[V]} \xi=\left[\begin{array}{cc}
1-2 \xi & 0 \\
-\frac{1}{2} \xi & 1-\frac{3}{2} \xi
\end{array}\right] \text { is regular for } \xi \in C^{-}:=\{\xi \in C \mid \operatorname{Re} \xi \leq 0\}, \\
\left(I_{2}-\sum_{V=1}^{2} A^{[V]} \xi\right)=\frac{\left[\begin{array}{cc}
1-(3 / 2) \xi(1 / 2) \xi \\
0 & 1-2 \xi
\end{array}\right]}{\left(1-(1 / 2) \xi+3 \xi^{2}\right)^{2}} \\
\sum_{V=1}^{2} A_{1}^{[V]^{T}} \xi\left(I_{2}-\sum_{V=1}^{2} A^{[V]} \xi\right)^{-1}=\frac{\left[2 \xi-3 \xi^{2}, \xi^{2}\right]}{\left(1-(7 / 2) \xi+3 \xi^{2}\right)^{2}} \\
\sum_{V=1}^{2} A_{2}^{[V]^{T}} \xi\left(I_{2}-\sum_{V=1}^{2} A^{[V]} \xi\right)^{-1}=\frac{\left[(1 / 2) \xi-(3 / 4) \xi^{2},(3 / 2) \xi-(11 / 4) \xi^{2}\right]}{\left(1-(7 / 2) \xi+3 \xi^{2}\right)}
\end{gathered}
$$

and (4.6b)-(4.6d) are uniformly bounded for $\xi \in C^{-}$,

$$
\begin{gathered}
R_{1}(\xi)=1+\sum_{V=1}^{2} A_{1}^{[V]^{T}} \xi\left(I_{2}-\sum_{V=1}^{2} A^{[V]} \xi\right)^{-1} e=\frac{\left(1-(3 / 2) \xi+\xi^{2}\right)}{\left(1-(7 / 2) \xi+3 \xi^{2}\right)} \\
R_{2}(\xi)=1+\sum_{V=1}^{2} A_{2}^{[V]^{T}} \xi\left(I_{2}-\sum_{V=1}^{2} A^{[V]} \xi\right)^{-1} e=\frac{\left(1-(3 / 2) \xi-(1 / 2) \xi^{2}\right)}{\left(1-(7 / 2) \xi+3 \xi^{2}\right)}
\end{gathered}
$$

and (4.6e) and (4.6f) satisfy that $\left|R_{i}(\xi)\right| \leq 1$, for $\xi \in C^{-}, i=1,2$.

By Theorems 2.4 and 3.7 we know that the ARKLM (2.1a), (2.1b), and (2.1c) corresponding to the method (4.4) is GDN stable and D-Convergent of order two. 


\section{Acknowledgments}

This paper was supported by the National Natural Science Foundation of China (11101109) and the Natural Science Foundationof Hei-long-jiang Province of China (A201107).

\section{References}

[1] T. E. Weldon, J. Kirk, and H. M. Finlay, "Cyclical granulopoiesis in chronic granulocytic leukemia: a simulation study," Blood, vol. 43, pp. 379-387, 1974.

[2] A. E. Ruehli, U. Miekkala, A. Bellen, and H. Heeb, "Stable time domain solutions for EMC problems using PEEC circuit models," in Proceedings of the IEEE International Symposium on Electromagnetic Compatibility, 1994.

[3] R. K. Brayton, "Small signal stability criterion for networks containing lossless transmission lines," IBM Journal of Research and Development, vol. 12, pp. 431-440, 1968.

[4] N. Guglielmi, "Inexact Newton methods for the steady state analysis of nonlinear circuits," Mathematical Models \& Methods in Applied Sciences, vol. 6, no. 1, pp. 43-57, 1996.

[5] Y. Kuang, Delay Differential Equations with Applications in Population Dynamics, vol. 191, Academic Press, Boston, Mass, USA, 1993.

[6] A. Bellen and M. Zennaro, Numerical Methods for Delay Differential Equations, Oxford University Press, New York, NY, USA, 2003.

[7] L. Torelli, "Stability of numerical methods for delay differential equations," Journal of Computational and Applied Mathematics, vol. 25, no. 1, pp. 15-26, 1989.

[8] L. Torelli, "A sufficient condition for GPN-stability for delay differential equations," Numerische Mathematik, vol. 59, no. 3, pp. 311-320, 1991.

[9] A. Bellen and M. Zennaro, "Strong contractivity properties of numerical methods for ordinary and delay differential equations," Applied Numerical Mathematics, vol. 9, no. 3-5, pp. 321-346, 1992.

[10] A. Bellen, "Contractivity of continuous Runge-Kutta methods for delay differential equations," Applied Numerical Mathematics, vol. 24, no. 2-3, pp. 219-232, 1997.

[11] M. Zennaro, "Contractivity of Runge-Kutta methods with respect to forcing terms," Applied Numerical Mathematics, vol. 11, no. 4, pp. 321-345, 1993.

[12] M. Zennaro, "Asymptotic stability analysis of Runge-Kutta methods for nonlinear systems of delay differential equations," Numerische Mathematik, vol. 77, no. 4, pp. 549-563, 1997.

[13] A. Araújo, "A note on B-stability of splitting methods," Computing and Visualization in Science, vol. 6, no. 2-3, pp. 53-57, 2004.

[14] B. García-Celayeta, I. Higueras, and T. Roldán, "Contractivity/monotonicity for additive RungeKutta methods: inner product norms," Applied Numerical Mathematics, vol. 56, no. 6, pp. 862-878, 2006.

[15] C. J. Zhang and S. Z. Zhou, "Nonlinear stability and D-convergence of Runge-Kutta methods for delay differential equations," Journal of Computational and Applied Mathematics, vol. 85, no. 2, pp. 225237, 1997.

[16] C. J. Zhang, S. Z. Zhou, and X. X. Liao, “D-convergence and GDN-stability of Runge-Kutta methods for a class of delay systems," Applied Numerical Mathematics, vol. 37, no. 1-2, pp. 161-170, 2001.

[17] R. Frank, J. Schneid, and C. W. Ueberhuber, "The concept of B-convergence," SIAM Journal on Numerical Analysis, vol. 18, no. 5, pp. 753-780, 1981.

[18] K. Dekker and J. G. Verwer, Stability of Runge-Kutta Methods for Stiff Nonlinear Differential Equations, vol. 2, North-Holland, Amsterdam, The Netherlands, 1984.

[19] J. D. Lambert, Numerical Methods for Ordinary Differential Systems, John Wiley \& Sons, Chichester, UK, 1991.

[20] W. H. Hundsdorfer, "Stability and B-convergence of linearly implicit Runge-Kutta methods," Numerische Mathematik, vol. 50, no. 1, pp. 83-95, 1986. 


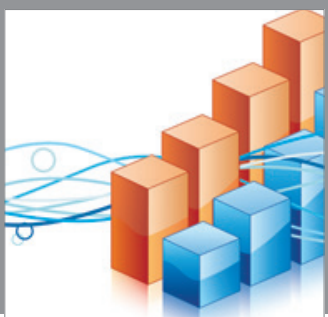

Advances in

Operations Research

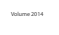

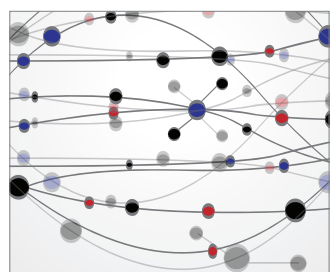

\section{The Scientific} World Journal
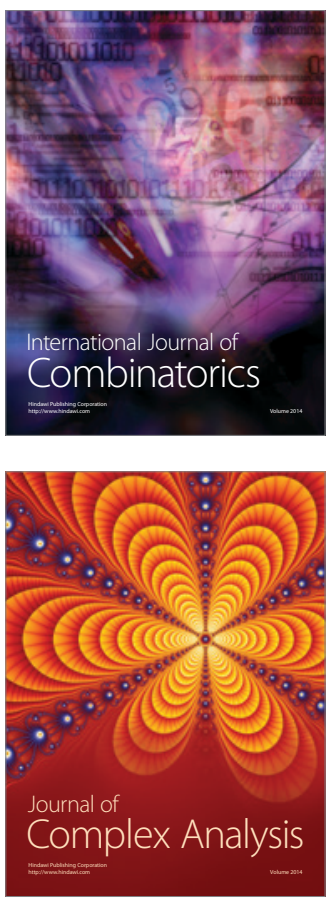

International Journal of

Mathematics and

Mathematical

Sciences
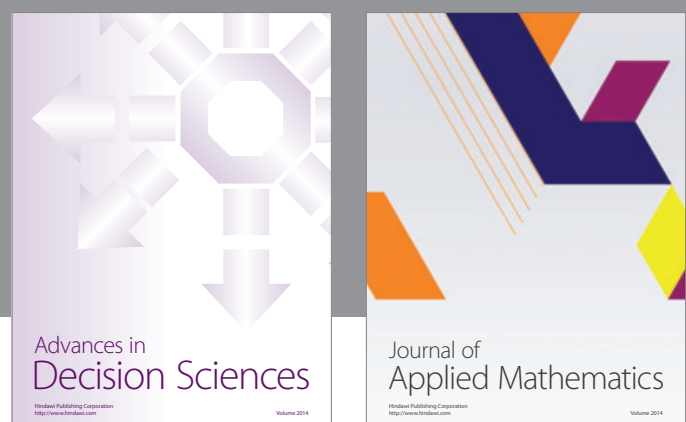

Journal of

Applied Mathematics
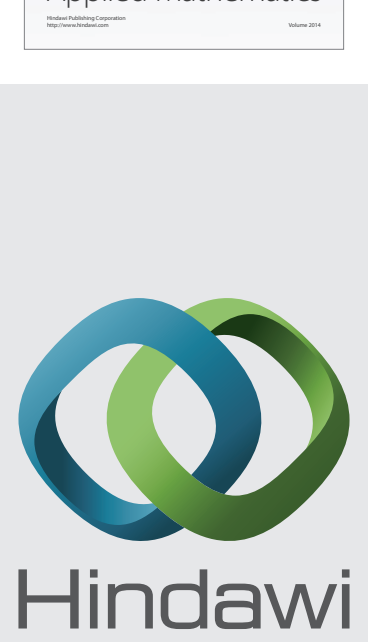

Submit your manuscripts at http://www.hindawi.com
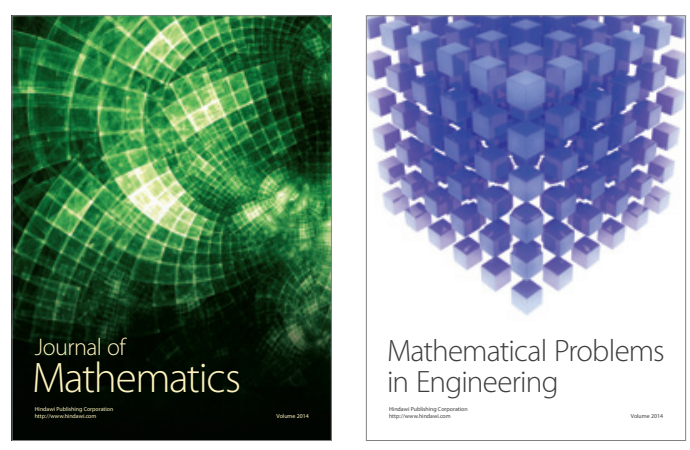

Mathematical Problems in Engineering
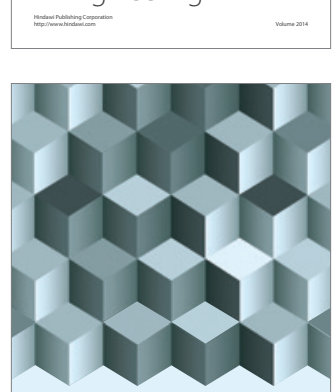

Journal of

Function Spaces
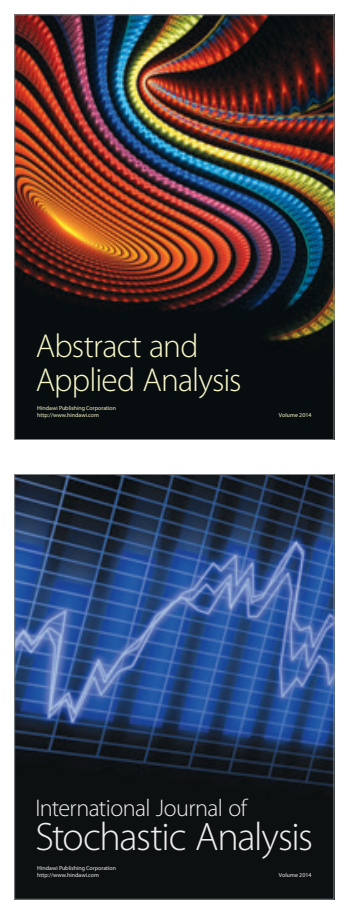

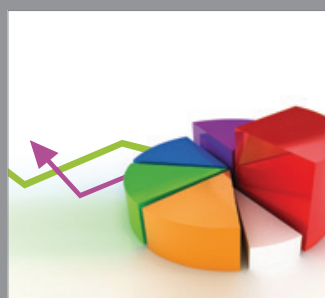

ournal of

Probability and Statistics

Promensencen
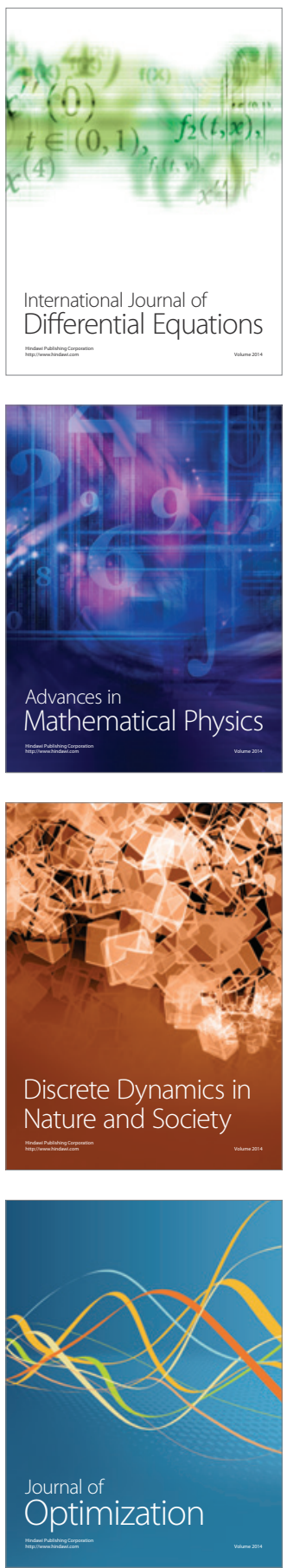